\title{
Optimizing decellularization techniques to create a new nerve allograft: an in vitro study using rodent nerve segments
}

\author{
Caroline A. Hundepool, PhD, ${ }^{1,2}$ Tim H. J. Nijhuis, MD, PhD, ${ }^{2}$ Dimitra Kotsougiani, MD, ${ }^{1}$ \\ Patricia F. Friedrich, AAS, ${ }^{1}$ Allen T. Bishop, MD, ${ }^{1}$ and Alexander Y. Shin, MD ${ }^{1}$ \\ 'Department of Orthopedic Surgery, Microvascular Research Laboratory, Mayo Clinic, Rochester, Minnesota; and 2Department \\ of Plastic, Reconstructive and Hand Surgery, Erasmus Medical Center, Rotterdam, The Netherlands
}

\begin{abstract}
OBJECTIVE Commercially available processed nerve allografts have been shown to be inferior to autografts in previous animal studies. The authors hypothesized that combining different processing and storage techniques will result in improved nerve ultrastructure preservation, lower immunogenicity, and minimized cellular debris. Different processing protocols were evaluated using chemical detergents, enzymes, and irradiation, with the addition the of enzyme elastase, were used. Additionally, the difference between cold and frozen storage was investigated. The goal of this study was to create an optimized nerve allograft.
\end{abstract}

METHODS Fifty rat nerves were decellularized with modifications of previous protocols and the addition of elastase. Subsequently, the nerve segments were stored at either $4^{\circ} \mathrm{C}$ or $-80^{\circ} \mathrm{C}$. Both processed and fresh control nerves were analyzed with confocal microscopy using immunohistochemical staining on the basal lamina (laminin $\gamma-1$ ), Schwann cells (S100 protein), and immunogenicity using major histocompatibility complex-I (MHCl) staining. Morphology of the ultrastructure and amount of cellular debris were analyzed on cross-sections of the nerves stained with toluidine blue and $\mathrm{H}$ $\& E$, and by using electron microscopy.

RESULTS Nerve ultrastructure was preserved with all decellularization protocols. Storage at $-80^{\circ} \mathrm{C}$ severely altered nerve ultrastructure after any decellularization method. Elastase was found to significantly reduce the immunogenicity and amount of Schwann cells, while maintaining good structural properties.

CONCLUSIONS Reduced immunogenicity, diminished cellular debris, and the elimination of Schwann cells was observed when elastase was added to the nerve processing while maintaining ultrastructure. Storage at $-80^{\circ} \mathrm{C}$ after the decellularization process heavily damaged the nerve ultrastructure as compared with cold storage. Further in vivo studies are needed to prove the nerve regenerative capacity of these optimized allografts.

https://thejns.org/doi/abs/10.3171/2017.1.FOCUS16462

KEY WORDS nerve allograft; processing; decellularization; elastase

$\mathrm{S}$ EGMENTAL peripheral nerve injures are devastating, life-altering injuries. ${ }^{11}$ Injuries with extensive damage require excision of the damaged nerve and a bridging segment (i.e., nerve graft) to provide a tensionfree nerve reconstruction. 6,15 To date, the nerve autograft represents the gold standard for reconstruction of extensive nerve damage. ${ }^{6}$ Nerve autografts are accompanied by significant donor site morbidity. Limitations in crosssectional area, length, and supply preclude optimal reconstructive options and often require prioritization of reconstructive goals. ${ }^{10,13}$ Therefore, throughout decades of research, alternatives have been proposed, developed, and implemented. Within the arsenal available, the surgeon can now choose between synthetic biocompatible conduits, autologous nonnerve tissues (i.e., vein or muscle), processed allografts, and autologous nerves. ${ }^{6,7}$

One of the alternatives to bridge nerve gaps is with processed allografts. Reconstruction with the use of nerve allografts has the advantage of mitigating donor site morbidity as well as providing an unlimited supply and length

ABBREVIATIONS BL = basal lamina; $\mathrm{MHCl}=$ major histocompatibility complex-l; SB-16 = sulfobetaine- 16 .

SUBMITTED October 31, 2016. ACCEPTED January 6, 2017.

INCLUDE WHEN CITING DOI: 10.3171/2017.1.FOCUS16462. 
of graft. A processed nerve allograft should not induce an immune reaction. Reduction of graft immunogenicity can be accomplished by eliminating cellular constituents, resulting in a mild to absent immune reaction. ${ }^{13,14}$ Remaining debris will also reduce or inhibit nerve regenerative capacity because debris may result in a fibrotic intraluminal occlusive blockade. Further enhancement of the regenerative capacity of the graft can be accomplished by the preservation of native extracellular matrix. The overall goal of nerve decellularization is to remove all cellular elements except the basal lamina (BL) - thus removing any immunogenicity of the tissue, as illustrated in Fig. 1. Aggressive processing will diminish immunogenicity but will also alter the structural properties. The optimal balance between removing cellular remnants while maintaining structural properties needs to be determined.

The current available processing protocols have been studied extensively, and a standard processing protocol is available. Regularly used protocols comprise a complex algorithm of steps including chemical detergents, irradiation, and cryopreservation. ${ }^{8}$ The basics of this protocol are essentially the foundation of decellularization of nerves and have only been slightly modified by numerous investigators. ${ }^{12}$ Given the fact that current processed allograft studies (both experimental and clinical) demonstrate inferior results to autografts, we sought to determine if the addition of elastase to the processing would improve decellularization. ${ }^{5}$ Elastase is an enzyme that deconstructs proteins by disconnecting peptide junctions and breaking down elastin. It has been successfully used in the decellularization of tissues other than nerves-for instance in heart valve and cartilage decellularization. ${ }^{16,17}$ Currently all the clinically available allograft conduits are frozen before use. In addition to optimizing the decellularization process, the effect of frozen storage versus cold storage on the efficacy of processed nerve grafts has not been adequately evaluated.

The purpose of this in vitro study was 2-fold. First, to study the effect of elastase on the decellularization of rat nerve tissue, and second to evaluate the effect of storage, either frozen or cold. We hypothesized that the addition of elastase will allow the extracellular matrix to be preserved with a minimal amount of debris. Determination of the optimal storage technique (e.g., cold or frozen) in addition to optimization of the decellularization process would allow for the optimal nerve allograft for the reconstruction of large peripheral nerve defects.

\section{Methods}

After approval by our institution's animal care and use committee, 25 Sprague-Dawley rats weighing 250-350 g (Harlan) were used. After initial isoflurane induction, all animals were killed with an overdose of pentobarbital. Bilateral $15-\mathrm{mm}$ nerve segments of the sciatic nerve were aseptically harvested. A total of 50 nerve segments were collected.

\section{Experimental Design}

A total of 5 groups were compared in this study. All groups consisted of 10 nerves. The first group was pro-
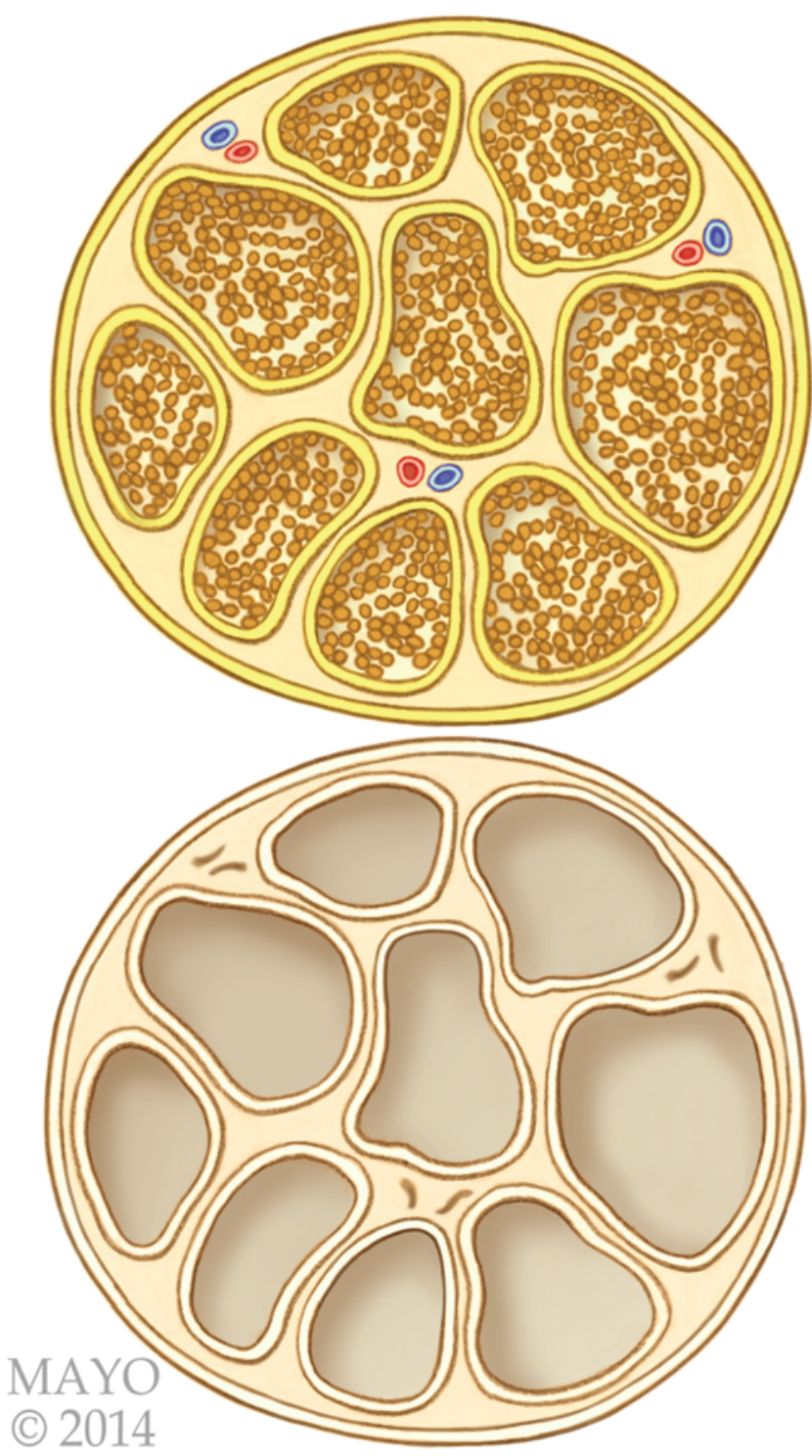

FIG. 1. Illustrations showing nerve graft (upper) and hypothesized nerve allograft (lower). The ultimate goal of processing a nerve allograft is to remove all cells and cellular debris, which are targeted in an immune response while preserving the extracellular matrix. Used with permission of Mayo Foundation for Medical Education and Research. All rights reserved.

cessed following the standard protocol based on previous studies. ${ }^{5,8,12}$ The second and third groups underwent the same processing, only with the addition of the enzyme elastase in 2 different time periods (i.e., 8 and 16 hours) (Groups II and III). The effect of frozen storage $\left(-80^{\circ} \mathrm{C}\right)$ was also studied in Group 4. A native unprocessed nerve (Group 5) was analyzed as a negative control. An overview of the studied groups is depicted in Table 1.

\section{Nerve Allograft Processing}

Briefly, immediately after harvest the nerve segments were placed in RPMI (Roswell Park Memorial Institute medium) 1640 solution at $4^{\circ} \mathrm{C}$ overnight. The next day the nerve tissues were placed in deionized distilled water. Af- 
TABLE 1. Experimental design for nerve allograft investigation

\begin{tabular}{cll}
\hline Group & \multicolumn{1}{c}{ Treatment } & \multicolumn{1}{c}{ Storage } \\
\hline I & Standard & Cold $\left(4^{\circ} \mathrm{C}\right)$ \\
\hline II & Standard + elastase (short) & Cold $\left(4^{\circ} \mathrm{C}\right)$ \\
\hline III & Standard + elastase (long) & Cold $\left(4^{\circ} \mathrm{C}\right)$ \\
\hline IV & Standard + elastase (long) & Frozen $\left(-80^{\circ} \mathrm{C}\right)$ \\
\hline V & Unprocessed native nerve & No \\
\hline
\end{tabular}

ter 8 hours, the water was replaced by a solution containing $125 \mathrm{mM}$ sulfobetaine-10 (SB-10), $10 \mathrm{mM}$ phosphate, and $50 \mathrm{mM}$ sodium. The nerves were agitated for 15 hours and rinsed for 15 minutes in a washing solution of $10 \mathrm{mM}$ phosphate and $50 \mathrm{mM}$ sodium. Next, the washing solution was replaced by a solution containing $0.14 \%$ Triton X-200, $0.6 \mathrm{mM}$ sulfobetaine-16 (SB-16), $10 \mathrm{mM}$ phosphate, and $50 \mathrm{mM}$ sodium, and agitated for 24 hours. Next, the tissues were rinsed with the washing solution $(50 \mathrm{mM}$ phosphate and $100 \mathrm{mM}$ sodium). The washing solution was replaced by SB-10 solution, and the nerves were agitated for 8 hours. Next, they were washed once using the washing solution and put into a solution of SB-16/Triton X-200. The nerves were agitated for 15 hours and then washed in a solution containing $10 \mathrm{mM}$ phosphate and $50 \mathrm{mM}$ sodium. Subsequently, nerves were incubated in a solution containing $2 \mathrm{U} / \mathrm{ml}$ chondroitinase ABC (avidin-biotin complex) for 16 hours at room temperature and then washed in a solution containing $10 \mathrm{mM}$ phosphate and $50 \mathrm{mM}$ sodium. In Groups II-IV the nerves, which underwent elastase treatment, were incubated in a solution containing $0.05 \mathrm{U} / \mathrm{ml}$ elastase at $37^{\circ} \mathrm{C}$ for 8 hours (Group II) or 16 hours (Groups III and IV). After that, the nerves were sterilized with a $2.5-\mathrm{kGy}$ dose of gamma radiation.

\section{Tissue Storage}

As previously described and as has been successful in other fields, tissue was stored frozen. ${ }^{6}$ Nerve segments were stored in Ringer solution at $-80^{\circ} \mathrm{C}$ for the frozen storage (Group IV) for a duration of 2 weeks before final analysis. The other storage method was cold storage $\left(4^{\circ} \mathrm{C}\right)$, in which nerves were placed in phosphate-buffered saline solution.

\section{Outcome Analysis \\ Structure}

A 5-mm section of each nerve segment was fixed in $2 \%$ Trump solution (37\% formaldehyde and $25 \%$ glutaraldehyde). Then, 1- $\mu \mathrm{m}$ thin sections were transversally cut and stained with $1 \%$ toluidine blue. Another 5-mm section of each nerve segment was suspended in OCT (optimal cutting temperature) compound, fast frozen, and $5-\mu \mathrm{m}$ transverse sections were cut. Nerve sections were stained with H \& E. Digital images of each sample were taken using a microscope digital camera (digital microscope color camera, 4.0 megapixels; Nikon). The organization of the BL was visualized with a laminin staining procedure as described below. For electron microscopy, ultrathin sections were cut (500 ̊), placed on copper grids (200 mesh, EMS), and stained with uranyl acetate and lead citrate (both from EMS). Sections were examined under a JEOL
1400 transmission electron microscope (JEOL Ltd.). All sections were scored for their structural properties on a 5 -point scale, with 1 being worst and 5 being optimal. The structure of the nerve segments was compared with the native, unprocessed nerve. Three independent and blinded investigators performed the analysis. Validity and reliability of the objective analysis was determined, with an intraclass correlation of 0.83 (95\% CI 0.71-0.90).

\section{Remnants (Axons and Immunogenicity)}

Intraluminal remnants were examined with immunohistochemical staining on different components of the nerve allograft. Nerve segments were prefixed with $4 \%$ cold paraformaldehyde and fast frozen. Transverse frozen sections (5- $\mu \mathrm{m}$ thickness) were cut. To identify the remnant axons left in the graft, S100 staining was performed. To study the immunogenicity of the graft after processing, major histocompatibility complex-I (MHCI) was stained. The combination of the presence of S100 and MHCI after nerve processing was classified as remnants and cellular debris. Additionally, laminin was stained to identify the basal laminae. The immunohistochemical staining procedure was performed using the Leica Bond III Stainer (Leica Microsystems). The sections were postfixed in $4 \%$ paraformaldehyde and retrieved online using Epitope Retrieval 1 (Leica Microsystems) for 5 minutes.

The primary antibodies used were as follows: polyclonal S100 anti-rabbit (Dako) was used at 1:5000; polyclonal laminin y1 anti-rabbit (Sigma) was used at 1:200; and mouse anti-MHCI (Clone OX18, Novus Biological) was used at 1:100. All antibodies were incubated for 60 minutes. The detection system used was the Research Detection System (Leica DS9455). This system includes the Protein Block (Dako X0909) and secondary antibody AlexFluor488. All sections were nuclear-stained with Hoechst 33342 (Invitrogen H1399). Once completed, slides were removed from the stainer and rinsed for 5 minutes in distilled water. Slides were coverslipped using ProLong Gold antifade media (Invitrogen). Nerve slides were examined under a fluorescence laser confocal microscope (LSM 780, Zeiss) and pictures were captured with a camera. The intensity of staining in the cross-section of the nerve was measured with ImageJ software (NIH) to quantify results of the different staining processes.

\section{Statistical Analysis}

Data are expressed as the mean \pm SEM. For structural analysis, the results of the 3 different observers and the 3 different staining processes were averaged to score the structural properties. Statistical analysis of the differences among the groups was performed with 1-way ANOVA, followed by the Bonferroni post hoc test with GraphPad Prism 5 software (GraphPad Software), and p values < 0.05 were considered to be significant.

\section{Results}

An overview of the different staining results is depicted in Fig. 2, and a summary of the results is given in Table 2. The results are graphically displayed in Figs. 3-5 and presented in Table 3. 


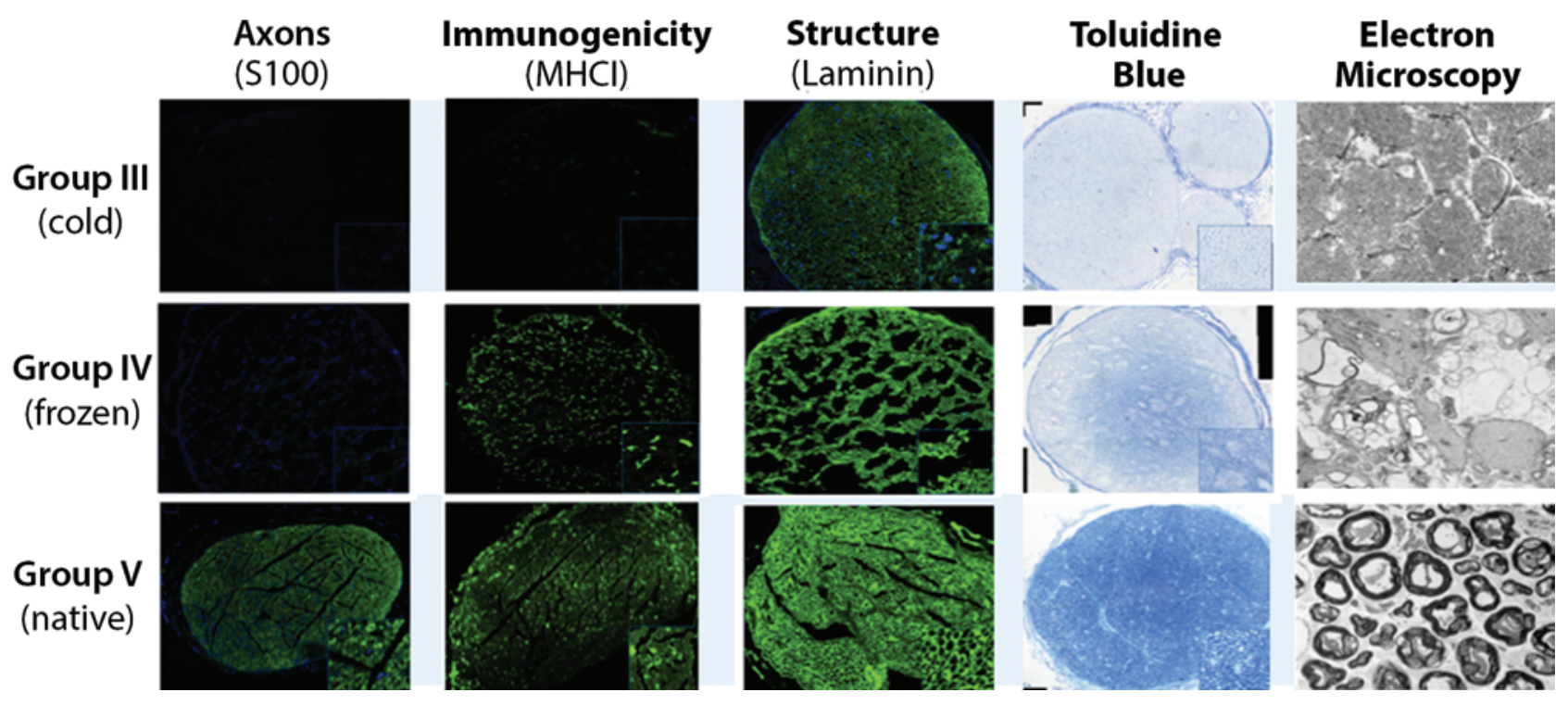

FIG. 2. Overview of the different staining materials used for Groups III, IV, and V.

\section{Enzymatic Decellularization \\ Structure: Score}

The structure of the nerve graft was not significantly influenced by the addition of elastase to the decellularization protocol. Group I, the standard protocol, showed a score of $3.9 \pm 0.2$, and in Group II, with the addition of elastase, the score was $3.5 \pm 0.1$. There was no significant difference between the groups. A longer exposure of the enzyme, Group III, did not influence the structure of the nerve graft $(3.2 \pm 0.1)$. There was no statistical difference between the groups (Fig. 3).

\section{Structure: Laminin}

The same was observed when the intensity of the laminin was determined. The enzyme did not significantly reduce the presence of laminin in the nerve. The nerves in Group I (standard protocol) had a laminin intensity of $65.7 \pm 8.6$; Group II (with elastase) $53.22 \pm 6.2$; and the nerves with a longer exposure to elastase, Group III, had a score of $51.5 \pm 3.3$. No significant difference among those 3 groups was observed $(\mathrm{p}=0.20)$.

\section{Remnants: Axons}

Axons were significantly reduced by the addition of the

TABLE 2. Summary of the effect of elastase and frozen storage on different components of the processed rat nerve segments

\begin{tabular}{lccc}
\hline Component & Source & Elastase & Frozen Storage \\
\hline Structure & & & \\
\hline Structural score & Rat & $=$ & $\downarrow$ \\
\hline Laminin & Rat & $=$ & $=$ \\
\hline Remnants & & & \\
\hline Axons & Rat & $\downarrow$ & $\uparrow$ \\
\hline Immunogenicity & Rat & $\downarrow$ & $\uparrow$ \\
\hline
\end{tabular}

Downward-pointing arrows denote decreased values; upward-pointing arrows denote increased values; and equals signs denote stable values. extra enzymatic step (Group I, $9.5 \pm 1.0$; Group II, $5.0 \pm$ $0.5)$. Prolonged exposure to elastase (Group III) showed an even lower score of axons (3.2 \pm 0.4$)$. The difference between the groups was statistically significant $(\mathrm{p}<0.0001)$ (Fig. 4).

\section{Remnants: Immunogenicity}

Elastase had a similar significant effect of reduction of the remnants when evaluating immunogenicity. The standard protocol without the enzyme (Group I) had an MHCI score of $18.2 \pm 1.8$; the addition of elastase reduced the MHCI score to $9.3 \pm 1.0$. The observed differences were significant $(\mathrm{p}=0.0010)$ (Fig. 5).

\section{Storage Effects}

Structure: Score

The effect of cold or frozen storage at either $4^{\circ} \mathrm{C}$ or $-80^{\circ} \mathrm{C}$ showed a tremendous effect on the structure of the

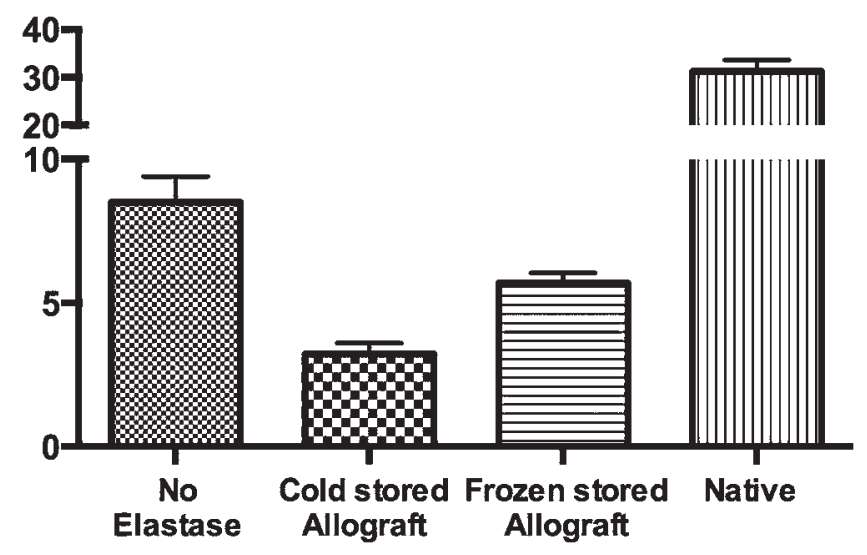

FIG. 3. Bar graph showing staining for axons. The axons, visualized with S100 staining, show a decrease in Groups III and IV when treated with elastase. Increments on the y axis denote the intensity of staining. Error bars denote the SEM. 


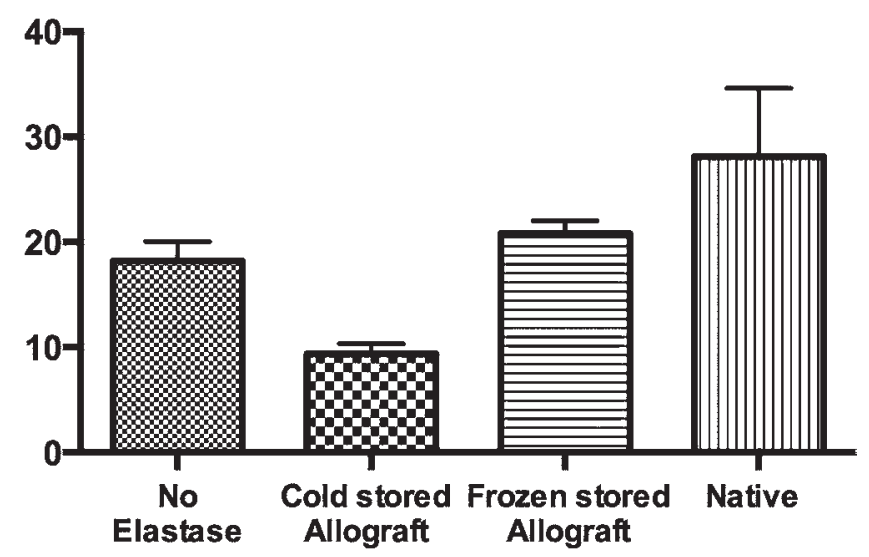

FIG. 4. Bar graph showing staining for immunogenicity. The immunogenicity of the nerve grafts, visualized with $\mathrm{MHCl}$ staining, shows a decrease with addition of elastase. Increments on the y axis denote the intensity of staining. Error bars denote the SEM.

graft. When frozen, the total score for the structure significantly decreased, from $3.2 \pm 0.1$ (Group III) to $1.6 \pm 0.2$ in Group IV for the rat nerves. The effect of storage was visualized with electron microscopy in Fig. 6.

\section{Structure: Laminin}

Storage had no significant effect on the laminin intensity staining. The score of Group III $(51.49 \pm 3.31)$ was not significantly different compared with the frozen group (IV) $(63.67 \pm 2.4, \mathrm{p}=0.20)$.

\section{Remnants: Axons}

When looking at the effect of storage on the intraluminal remnants, it was demonstrated that the nerves kept in cold storage contained a significantly lower amount of axons stained with S100 compared with the frozen nerves. The effect of storage was statistically significantly different. Group III (3.2 \pm 0.4$)$ was the cold-storage group; the values for frozen storage (Group IV) were higher (5.7 \pm 0.3) (Fig. 4).

\section{Remnants: Immunogenicity}

The effect of cold or frozen storage at either $4^{\circ} \mathrm{C}$ or $-80^{\circ} \mathrm{C}$ showed a severe effect on the structure of the graft. The immunogenicity in the rat nerves of the cold storage (Group III, stained with MHCI) was statistically significantly different $(9.3 \pm 1.0)$ from the nerves kept in frozen storage (Group IV, $20.8 \pm 1.2$ ), as depicted in Fig. 5.

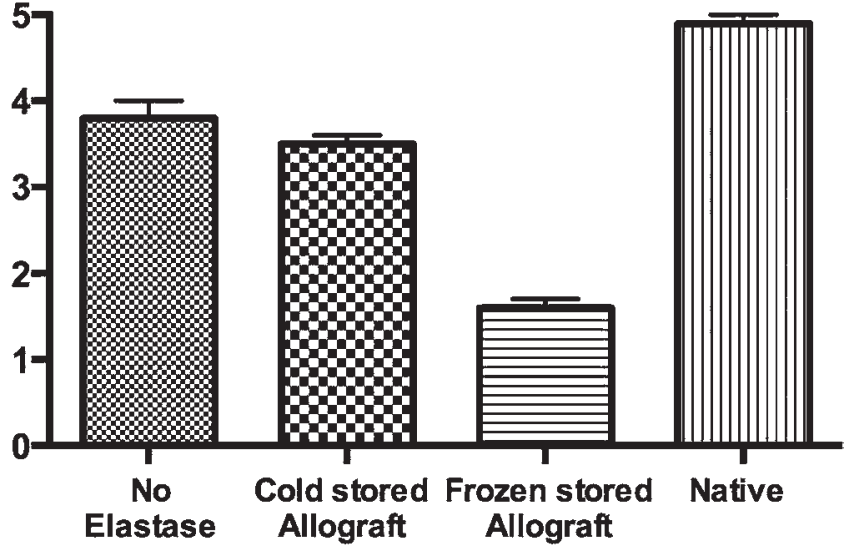

FIG. 5. Bar graph showing staining for structure. The structure score of the nerve grafts was not significantly influenced by the enzyme. Frozen storage of the grafts significantly reduced the score, which ranged from 1 (worst) to 5 (optimal).

\section{Discussion}

This study demonstrated that the enzyme elastase reduced immunogenicity, diminished cellular debris, and cleared Schwann cells better-while maintaining the ultrastructure-compared with standard decellularization protocols. Storage at $-80^{\circ} \mathrm{C}$ after the decellularization process heavily damaged the nerve ultrastructure compared with cold storage. Currently available nerve allograft processing techniques are not sufficient to create nerve allografts that can truly challenge the nerve autograft in the clinical setting. Creating a nerve allograft has therefore been the subject of multiple studies. $3 ., 8,12,14$

Knowledge of the ultrastructural anatomy of a peripheral nerve is key to understanding the concept of nerve allograft transplantation. There are 2 domains in the ultrastructure: an internal BL, and an external fibrillar matrix. The BL faces and is linked to cellular membranes, and its components typically include laminin heterotrimers, collagens, nidogen or entactin, and poreoglycans. The exact molecular assortment of the BL is cell-specific. ${ }^{2}$ Schwann cells, undoubtedly, are essential for myelination and are responsible for the fast-conducting signaling. It has been shown that preservation of the BL is necessary to support the host Schwann cells and to direct new axon sprouts. ${ }^{9}$ Without laminin, Schwann cells cannot differentiate into a myelinating phenotype. ${ }^{1}$ Their relation is essential for a structured conducting pattern. Previous studies have shown that in mice lacking the laminin $\gamma-1$ gene, axon

TABLE 3. Results showing the effect of elastase and frozen storage

\begin{tabular}{lccccc}
\hline \multicolumn{1}{c}{ Component } & $\begin{array}{c}\text { Group I } \\
\text { (no elastase) }\end{array}$ & $\begin{array}{c}\text { Group III } \\
\text { (cold-stored allograft) }\end{array}$ & $\begin{array}{c}\text { Group IV } \\
\text { (frozen stored allograft) }\end{array}$ & $\begin{array}{c}\text { Effect of Elastase; } \\
\text { p Value }\end{array}$ & $\begin{array}{c}\text { Effect of Storage; } \\
\text { p Value }\end{array}$ \\
\hline Axons & $9.5 \pm 1.0$ & $3.2 \pm 0.4$ & $5.7 \pm 0.3$ & $<0.0001$ & 0.0003 \\
\hline Immunogenicity & $18.2 \pm 1.8$ & $9.3 \pm 1.0$ & $20.8 \pm 1.2$ & 0.0010 & $<0.0001$ \\
\hline Structure & $3.9 \pm 0.2$ & $3.2 \pm 0.1$ & $1.6 \pm 0.2$ & $\mathrm{NS}$ & 0.0013 \\
\hline
\end{tabular}

NS $=$ not significant .

Values (mean $\pm \mathrm{SD}$ ) are presented as follows: number of axons; the $\mathrm{MHCl}$ score for immunogenicity; and scores based on a scale ranging from 1 (worst) to 5 (optimal) for structure. 

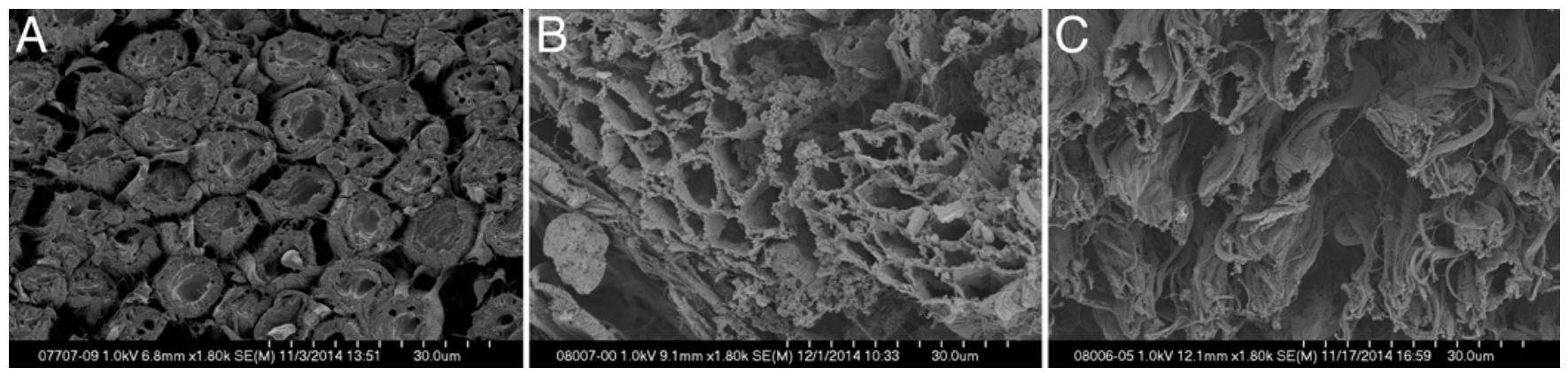

FIG. 6. Scanning electron microscopic images of the nerve graft. The images show native nerve: Group V (A); cold-preserved nerve allograft: Group II (B); and frozen nerve allograft: Group III (C).

sorting and myelination is derailed, affecting the capacity of axons to regenerate. ${ }^{1}$ In addition to its presence, the organization of the basal membrane is of paramount importance in supporting regeneration. Fragmentation of the BL has been demonstrated using electron microscopy after chronic denervation. The progressive fragmentation of the basal membrane is negatively correlated with the reinnervation. ${ }^{4}$ Therefore, evaluating the integrity of the basal membrane is of fundamental importance to determine the ideal processing technique to create a nerve allograft.

This study demonstrates the effect of modifications to the standard processing techniques in a rat model. Modifications to the processing protocol should not disrupt the presence and organization of the BL, but should reduce the remnant debris and axons. The standard processing technique was modified by addition of an extra enzymatic step. The enzyme elastase was hypothesized to remove more of the remnants of cells and debris by disrupting the tight junctions. ${ }^{16}$ In this study both the organization and the presence of laminin was investigated. When evaluating the amount of axons remaining in the nerve graft, the addition of elastase showed a significant reduction of S100 expression. Longer exposure to the enzyme did not significantly further reduce the cellular remnants in the rat nerves. The immunogenicity of the grafts was also reduced by the addition of the enzyme. The elastase-processed nerves provided a nerve allograft with reduced cellular remnants and preservation of the ultrastructure.

The addition of elastase reduced the amount of axons in the nerve allograft by $66.3 \%$. The immunogenicity was reduced by $48.9 \%$ when the enzyme was added to the decellularization process. By frozen storage of the nerve allograft, the structural score was reduced by $50 \%$ when compared with the cold-stored allograft. The effect of both the elastase and storage on the allograft was significantly different.

Previously produced nerve allografts were preserved at $-80^{\circ} \mathrm{C} .^{17}$ In other research fields (bone allograft studies), the allograft structure is less fragile than the nerve tissues and tolerates preservation by freezing better than nerve tissue. ${ }^{17}$ The destructive effect of freezing on nerve tissues was found in our study. The processed nerve allograft that was stored frozen proved to have significantly worse structure compared with the nerves for which the cold-storage preservation method was used. In Fig. 3 the difference between the 2 storage methods studied is demonstrated. It can be appreciated that the ultrastructure of the nerve, still visible in the cold-stored nerve, is severely deconstructed in the nerves that were stored frozen. The structure can almost no longer be observed. The significant difference observed with the structural scoring was not present in the laminin staining. The presence of laminin did not significantly differ between cold- and frozen-storage groups. However, the structural scoring and preservation of the BL showed big differences.

We recognize the limitations of this study, in particular that the effect of the processing is only investigated in vitro. The neuroregenerative effect of our proposed processing and storage of the allograft still needs to be studied in an in vivo animal model. In this study the immunogenicity of the processed nerve segments was investigated with MHCI staining. The true immune response to the processed nerve allografts should be investigated in vivo. Furthermore, it could be argued that more groups need to be investigated. For instance, different temperatures could be incorporated in this study. We choose to use 2 temperatures $\left(4^{\circ} \mathrm{C}\right.$ and $\left.-80^{\circ} \mathrm{C}\right)$ because these are the most commonly used temperatures in the clinical setting. To maintain power for this study we only used 5 different groups.

\section{Conclusions}

The aforementioned limitations notwithstanding, we have clearly demonstrated that the addition of the enzyme elastase to nerve processing resulted in maintenance of the nerve ultrastructure while significantly reducing immunogenicity, and diminishing cellular debris and Schwann cells, compared with standard decellularization protocols. Additionally, we demonstrated that storage at $-80^{\circ} \mathrm{C}$ after the decellularization process heavily damaged the nerve ultrastructure, as compared with cold storage at $4^{\circ} \mathrm{C}$. We have been able to improve the ultrastructure while further reducing the immunogenic capacity of the nerve allografts in this study by the addition of the enzyme elastase and modification of storage after nerve processing. Further research should focus on an in vivo study of the neuroregenerative effect of nerve allografts optimized by this means.

\section{References}

1. Chen ZL, Strickland S: Laminin $\gamma 1$ is critical for Schwann cell differentiation, axon myelination, and regeneration in the peripheral nerve. J Cell Biol 163:889-899, 2003

2. Court FA, Wrabetz L, Feltri ML: Basal lamina: Schwann 
cells wrap to the rhythm of space-time. Curr Opin Neurobiol 16:501-507, 2006

3. Fox IK, Jaramillo A, Hunter DA, Rickman SR, Mohanakumar T, Mackinnon SE: Prolonged cold-preservation of nerve allografts. Muscle Nerve 31:59-69, 2005

4. Giannini C, Dyck PJ: The fate of Schwann cell basement membranes in permanently transected nerves. J Neuropathol Exp Neurol 49:550-563, 1990

5. Giusti G, Willems WF, Kremer T, Friedrich PF, Bishop AT, Shin AY: Return of motor function after segmental nerve loss in a rat model: comparison of autogenous nerve graft, collagen conduit, and processed allograft (AxoGen). J Bone Joint Surg Am 94:410-417, 2012

6. Griffin JW, Hogan MV, Chhabra AB, Deal DN: Peripheral nerve repair and reconstruction. J Bone Joint Surg Am 95:2144-2151, 2013

7. Houdek MT, Shin AY: Management and complications of traumatic peripheral nerve injuries. Hand Clin 31:151-163, 2015

8. Hudson TW, Liu SY, Schmidt CE: Engineering an improved acellular nerve graft via optimized chemical processing. Tissue Eng 10:1346-1358, 2004

9. Ide C, Osawa T, Tohyama K: Nerve regeneration through allogeneic nerve grafts, with special reference to the role of the Schwann cell basal lamina. Prog Neurobiol 34:1-38, 1990

10. IJpma FF, Nicolai JP, Meek MF: Sural nerve donor-site morbidity: thirty-four years of follow-up. Ann Plast Surg 57:391-395, 2006

11. Jaquet JB, Luijsterburg AJ, Kalmijn S, Kuypers PD, Hofman A, Hovius SE: Median, ulnar, and combined median-ulnar nerve injuries: functional outcome and return to productivity. J Trauma 51:687-692, 2001

12. Neubauer D, Graham JB, Muir D: Chondroitinase treatment increases the effective length of acellular nerve grafts. Exp Neurol 207:163-170, 2007

13. Shin AY, Spinner RJ, Steinmann SP, Bishop AT: Adult traumatic brachial plexus injuries. J Am Acad Orthop Surg 13:382-396, 2005

14. Sondell M, Lundborg G, Kanje M: Regeneration of the rat sciatic nerve into allografts made acellular through chemical extraction. Brain Res 795:44-54, 1998

15. Sunderland S: Factors influencing the course of regeneration and the quality of the recovery after nerve suture. Brain 75:19-54, 1952
16. Tedder ME, Simionescu A, Chen J, Liao J, Simionescu DT: Assembly and testing of stem cell-seeded layered collagen constructs for heart valve tissue engineering. Tissue Eng Part A 17:25-36, 2011

17. Utomo L, Pleumeekers MM, Nimeskern L, Nürnberger S, Stok KS, Hildner F, et al: Preparation and characterization of a decellularized cartilage scaffold for ear cartilage reconstruction. Biomed Mater 10:015010, 2015

\section{Disclosures}

The authors report no conflict of interest concerning the materials or methods used in this study or the findings specified in this paper. This study was funded by Mayo Clinic Regenerative Medicine (Established Investigator Award); University of Montana; Michael van Vloten Foundation; and Trustfonds, Erasmus University, Rotterdam.

\section{Author Contributions}

Conception and design: Shin, Hundepool, Nijhuis, Kotsougiani, Bishop. Acquisition of data: Hundepool, Kotsougiani, Friedrich. Analysis and interpretation of data: Shin, Hundepool, Nijhuis, Kotsougiani, Bishop. Drafting the article: Hundepool, Kotsougiani. Critically revising the article: Shin, Hundepool, Nijhuis, Friedrich, Bishop. Reviewed submitted version of manuscript: Shin, Nijhuis, Friedrich, Bishop. Statistical analysis: Hundepool. Administrative/technical/material support: Friedrich. Study supervision: Shin, Bishop.

\section{Supplemental Information \\ Previous Presentations}

Parts of this study were presented at the annual meeting of the American Society of Peripheral Nerve (ASPN) held in the Bahamas in January 2015, and at the 70th annual meeting of the American Society of Surgery of the Hand (ASSH) held in 2015 in Seattle, Washington.

\section{Correspondence}

Alexander Y. Shin, Department of Orthopedic Surgery, Mayo Clinic, 200 1st St. SW, Rochester, MN 55905. email: shin. alexander@mayo.edu. 